\title{
Improving the execution speed of compiled Prolog with modes, clause selection, and determinism
}

\author{
Peter Van Roy $\uparrow$ \\ Katholieke Universiteit Leuven \\ Departement Computerwetenschappen \\ Celestijnenlaan 200A \\ B-3030 Heverlee \\ Belgium \\ Bart Demoen \\ BIM \\ Kwikstraat 4 \\ B-3078 Everberg \\ Belgium \\ Yves D. Willems \\ Katholieke Universiteit Leuven \\ Departement Computerwetenschappen \\ Celestijnenlaan 200A \\ B-3030 Heverlee \\ Belgium
}

\section{Introduction}

Much Prolog code is deterministic or almost so. The searching capabilities of Prolog are only used in special situations. Many Prolog procedures consist of a number of clauses of which only one (or a few) can succeed at any call. In fact, some are in effect case statements. Too of ten this code is executed by the general mechanism of backtracking which has much overhead [Mellish 85]. But the back tracking performed in most cases is but a special case. It is usually called shallow backtracking because it stays with the parent's choice point.

We describe a novel method of compiling Prolog which avoids the general backtracking mechanism whenever possible. In particular, it increases the speed of shallow backtracking and avoids the creation of choice points. It is developed with the BIM-Prolog system [Demoen 86] in mind, but any Prolog based on the 'Warren Abstract Machine' described in [Warren 83], can benefit from it. In this report a familiarity with the so called WAM is assumed.

The method is based on three concepts: modes, deterministic clauses, and improved clause indexing. Our goal is to make shallow backtracking as cheap as possible without hindering the programmer. Creating choice points and selecting clauses by unification and failure are replaced, as much as possible, by completely deterministic testing and selection of clauses. The aim is to achieve an efficiency comparable to that of simple tests and indexed jumps in conventional languages. This is realised by:

(1) A new scheme for clause indexing which is efficient and smart. It avoids creating choice points whenever possible. It gives the programmer the freedom to define predicates in any way without loss of efficiency. The compiler determines how to use the procedure's arguments for selection of clauses.

(2) Taking advantage of determinism in clauses. Clause indexing not always narrows the possibilities down to a single clause. In that case, the combination of unification and general backtracking is replaced by simple tests, jumps and a quick restoration of

$\dagger$ presently at the Computer Science Division, Department of EECS, University of Califomia at Berkeley, Berkeley, CA 94720. 
state. We introduce a class of clauses for which this technique is extremely fast. A definition of deterministic clauses, covering much Prolog code, is given.

(3) Taking advantage of mode declarations of procedures. The Prolog programmer should add mode declarations wherever maximal execution speed is needed. Our mode definitions combine flexibility for the programmer with usefulness for the compiler. Note that we obtain improvements even whithout mode declarations.

(4) An extension of the abstract machine's instruction set to support the above three optimizations. The instruction set of [Warren 83] is extended in a logical way to gain the maximum advantage from indexing, determinism, and modes. Keeping an intermediate code rather than moving straight to assembly makes the operation of the abstract machine much clearer. A translation to assembly should give no problems because the new instructions are simpler than existing instructions.

(5) A modified compilation scheme for clauses and procedures. The existing compiler serves as a base to implement the improved scheme. The new clause and procedure are compilers largely independent. The main disadvantage of the scheme is the increase in the size of the generated code.

(6) The optimization was developed with the concept of graceful degradation in mind. As fewer and fewer of the conditions for an optimal compilation are fulfilled, the performance improvement decreases gracefully. But it remains significant. The maximum speedup is obtained for a procedure consisting only of deterministic clauses, with distinct head arguments and annotated with modes. But the speedups obtained when one or more of these three conditions are missing from some clauses is still significant.

The use of modes to increase performance is well known [Warren 77]. But the net performance improvement due only to the resulting speedup of unification is difficult to measure. This is because modes allow for other optimizations. According to several Pro$\log$ implementors the net improvement is in the neighborhood of $10 \%$, but for nrev/2, the effect of modes is a factor of 3 in BIM-Prolog.

The performance improvement due to efficient clause indexing depends greatly on the particular procedure tested. Tests show that it reaches peaks of orders of magnitude for some search tasks.

Measurements were done to determine the performance improvements due to determinism alone. For a small procedure which can exploit it, a decrease in execution time of about $30 \%$ can be expected. This is the case for the procedure split/4 in Warren's quicksort benchmark [Warren 77]. An upper limit on the speed improvement is about a factor of 3. It is therefore clear that this optimization is promising.

The optimization of Prolog compilation has also been studied by Mellish [Mellish 85]. Especially the recognition of determinate predicates and the automatic generation of modes. Recognition of determinism is also addressed in [Sawamura \& Takeshima 85] and generalised to the notion of functionality in [Debray \& Warren 86a]. A better approach to automatic mode generation is in [Debray \& Warren $86 \mathrm{~b}$ ] and is further improved upon in [Bruynooghe et a1. 86]. The latter discusses some other optimizations based on information derived by abstract interpretation. That information on determinism and modes can help in closing the performance gap between Prolog and conventional languages is illustrated with a prototype of a very restricted language in [Bruynooghe \& Weemeeuw 86]; a broader discussion is in [Bruynooghe 86 ].

This paper is a shortened and improved version of [Van Roy \& Demoen 86]

\section{Definitions}

\subsection{Definition of a try-block}

A try-block is an ordered set of clauses which must be tried in sequence. It is usually implemented as a block of code starting with a try instruction, followed by retry instructions, and ending with a trust instruction: 


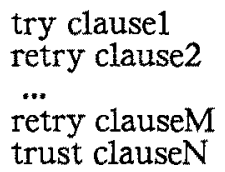

A try-block is a subset of the clauses of a procedure. The indexing algorithm of the compiler (section 4) attempts to reduce the set of clauses to be tried and passes control between clauses in a try-block by means of fast sequences of tests.

\subsection{Definitions and notations for modes}

In this section the rationale for the different modes is explained. Our goal is to obtain code consisting of a simple sequence of selections. Modes help in two ways: they tell which head arguments can be used to select against, and they help to determine if a clause is deterministic. We classify head arguments into four basic types. Either the programmer can tell the compiler the type of each argument, or the types can be inferenced automatically [Debray \& Warren 86b]. Our modes are inspired by [Warren 77] and [Mellish 81]. The most important difference is the introduction of structured modes. These mode definitions can be compiled easily.

\subsubsection{The basic modes}

Our basic modes are listed in table 1.

\begin{tabular}{|c|c|c|c|}
\hline \multicolumn{4}{|c|}{ Table 1: Notation for the basic modes } \\
\hline Mode & Synonyms & & Characterization \\
\hline Input & $i$ in & \pm & Main functor instantiated. \\
\hline Output & out & - & Unbound variable which does not share. \\
\hline Ground & $\mathrm{g}$ & ++ & Contains no variables. \\
\hline Input-output & inout & $?$ & All other cases. \\
\hline
\end{tabular}

\subsubsection{Structured modes}

To pinpoint more complex cases, structured modes are introduced. They are refinements of the input mode because they tell more about the main functor than mode input does. There are two structured modes.

$\mathrm{s}(\mathrm{M} 1, \mathrm{M} 2, \ldots, \mathrm{Mn})$

where $\mathrm{Mi}$ is any mode, and $\mathrm{n}$ is the arity. This mode is only used for clauses where the head argument is any structure (except a list) of arity $n$. This mode says that the argument is instantiated at run time to an n-ary structure whose ith component has mode Mi.

1(M1, M2)

where M1 and M2 are any modes. The actual argument must be a list, with the modes M1 and M2 for its head and tail.

\subsection{Definition of a test}

A procedure call $\mathrm{Q}$ in a procedure $\mathrm{P}:-\ldots, \mathrm{Q}, \ldots$ is a test if execution of $\mathrm{Q}$

(1) does not cause side-effects

(2) does not create choicepoints

(3) only binds variables local to clause $P$ and $Q$

(4) does not create structures on the heap.

In a similar spirit, unifications in the head of the procedure $P$ can be classified into test or non-tests, depending on whether they satisfy conditions (3) and (4). Q being a test is a dynamic notion because it depends on the instantiation pattern of the call to $P$. The class of tests can be extended in various ways by relaxing (3) and (4). In our current system, only calls to certain built-ins are recognised as tests. 


\subsection{Definition of a deterministic clause}

If, by executing only tests, control can reach a point in a clause - the commit point such that all following, yet untried clauses can be discarded without affecting the outcome of the computation (they will fail without side-effects) then that clause is a deterministic clause. Our notion of deterministic clause is very pragmatic, for a more principled one see [Debray \& Warren 86a]. Whether a clause is deterministic or not can depend on different factors:

(1) The clause itself. A cut in the body of the clause, preceded only by tests, makes the clause deterministic.

(2) On the procedure of which the clause is a part and its mode. If it is possible to show that the form of the input or ground arguments and the tests of a clause $\mathrm{C}$ are such that none of the following clauses can be entered beyond their tests, then inserting a cut af ter the tests of $\mathrm{C}$ does not alter in any way the meaning of the procedure. So $\mathrm{C}$ is a deterministic clause. The input-output and output arguments' values do not have any influence.

(3) The actual arguments of a call. They can be such that the following clauses cannot unify with them. For example, if an input argument is a constant, and all following clauses have a structure in the head, then the clause is deterministic for constants.

Example:

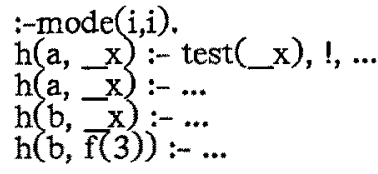

The first clause is deterministic because of the cut (1).

The second clause is deterministic because of (2): if the input argument matches ' $a$ ', it cannot match ' $b$ '. The second clause can be replaced by:

$$
h(a, \ldots):-!, \ldots
$$

The third clause is deterministic if the second argument at the call is a constant or a list (3). If the second argument is a structure then the third clause is not deterministic.

The fourth clause is deterministic because it is the last clause (2).

\subsection{Definition of the head of a deterministic clause}

If there exists a (real or imaginary) cut operator preceded by tests only, then the head of a deterministic clause is considered to include all tests up to the cut operation.

\section{Compilation of a single clause}

The method sketched in this section is an extension of the code generation for WAM in [Van Roy 84].

\subsection{The entry points}

We take advantage of deterministic clauses and delay the creation of a choice point. This requires extra entry points: a single clause can have up to four entry points.

\section{Determinism and nondeterminism of the clause}

A clause can be contained in many try-blocks. Whether or not a clause is deterministic depends on the particular try-block. Some clauses are always deterministic, for example those with a cut as first goal and only input arguments. Other clauses are only deterministic in some try-blocks. Being deterministic or not depends on different factors: the clause itself, all clauses in the procedure, the modes, and the try-block considered. See section 2.

Different actions are necessary in the two cases. A nondeterministic clause may need to create a choice point, whereas a deterministic clause never needs to do this. A clause therefore needs two entries: one where it is considered as deterministic, and one where it is nondeterministic. 


\section{The existence of a choice point}

At the entry of a clause, there may or may not be a choice point created by a previous clause of the same procedure. Different actions are necessary in these two cases as well. A comitted deterministic clause must remove the choice point if it exists. A nondeterministic clause has to change the retry address of the choice point if it exists. Otherwise it must set up the choice point itself. Therefore the two entries, deterministic and nondeterministic, must each be split into two parts: with or without a choice point.

\section{The four entry points}

(1) A deterministic entry with choice point (det-cp),

(2) A deterministic entry without choice point (det-nocp),

(3) A nondeterministic entry with choice point (nondet-cp),

(4) A nondeterministic entry without choice point (nondet-nocp).

deterministic entries

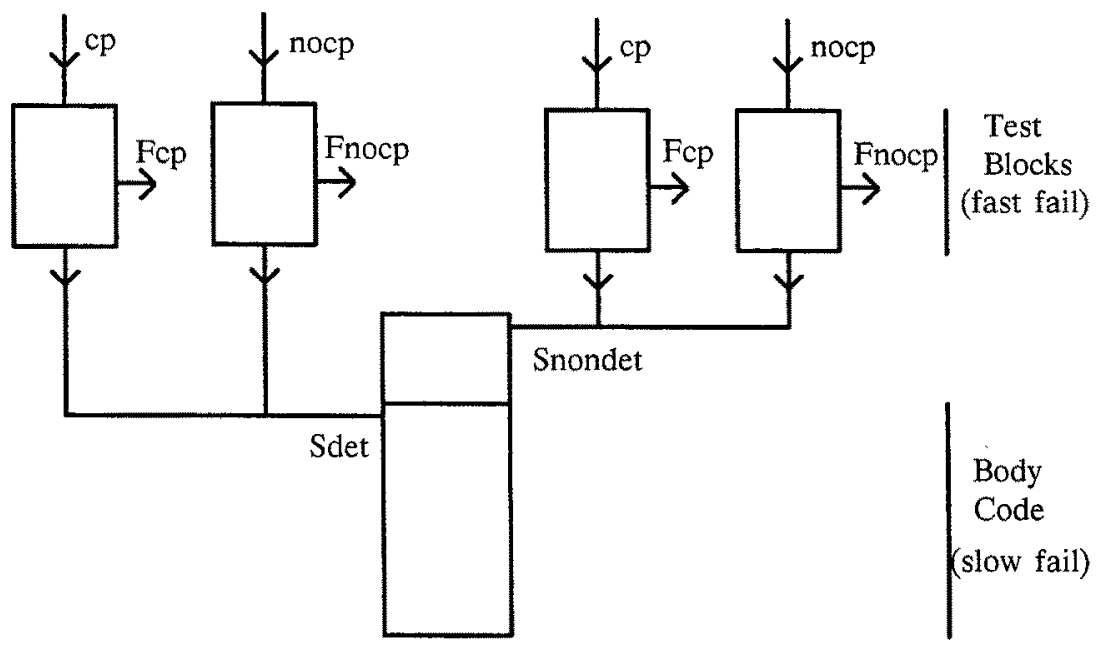

Figure 1 : Form of the clause code

\subsection{The clause code}

Figure 1 shows the internal structure of the clause code. Each entry has its own block of test code, which rapidly tests the success or failure of the clause. If the test succeeds then a jump is done to the body code, which is common to all clauses. Each test block has one failure exit, which jumps to the following clause in the try-block if the test fails.

\section{Kinds of failure}

As can be seen from figure 1, there are three kinds of failure. There are two basic kinds of failure:

(1) Fnocp: fast failure, no choice point: failure consists of restoring some registers and jumping to the nocp entry of the next clause (if there is no next clause, a special label initiates a slow failure). 
(2) Fcp : fast fail, choice point exists: failure consists of restoring some registers and jumping to the $c p$ entry of the next clause.

In the nondeterministic case, no registers need to be restored because of a peculiarity of the nondet test blocks. This, as well as the Sdet and Snondet entries in the body, will be clarified in section 3.3 and 3.4 .

(3) Slow failure : if the body fails, then the slow standard backtracking is initiated. The retry address is the $c p$ entry of the next clause.

deterministic

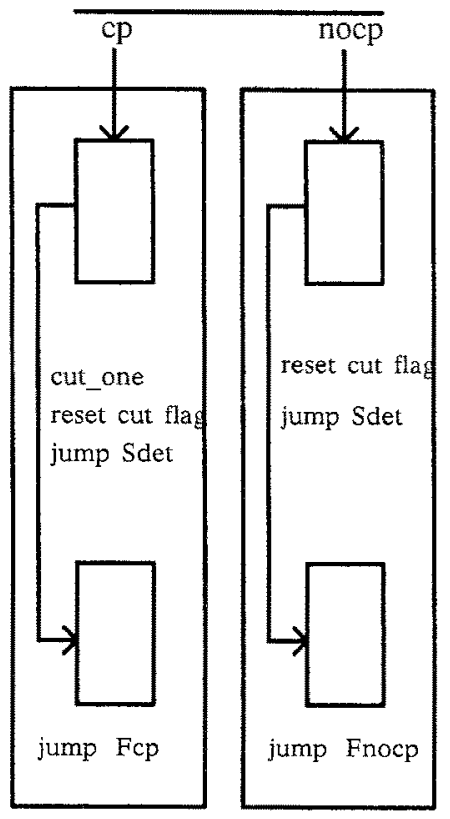

non deterministic

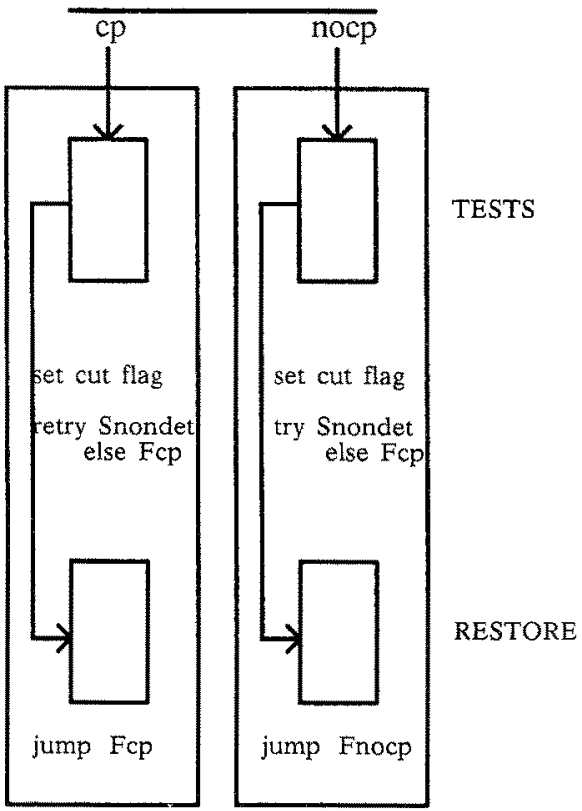

Figure 2 : Form of the test blocks

\subsection{Form of the test blocks}

In figure 2 the internal structure of each of the four test blocks is given. For nondeterministic entries, the notion of 'test' is rather restricted: it may not change any registers. This is because if the test code succeeds in the nocp case, a choice point must be created with all registers in their original state. The test code for deterministic entries may do bindings and unifications: upon failure the restore instructions will restore the state.

In the det-cp entry the instruction cut_one removes the choice point. The cut flag is not needed for this. The nondet-nocp entry is the only test block which can create a choice point. It does so with the 'try' instruction. It will jump to $F_{c p}$ if it created a choicepoint, otherwise to Fnocp. In the nondet-cp entry the 'retry' instruction updates the retry address of the existing choice point.

The cut flag is not needed in the test blocks since the presence or absence of the choice point is known upon entry. The cut flag is needed only for cuts in the body. If the body contains no cuts then it is not needed at all. If needed, it can be set or reset in the test blocks just before the body code is entered. 


\subsection{Form of the body code}

Consider the body code in figure 1. It has two entries from the det and nondet test blocks, denoted by the labels Snondet and Sdet. The reason for this is the difference in the tests which can be done by the det and nondet test blocks.

A nondet test block may not alter any registers. Therefore only a small part of the head is allowed in the test block. The other part of the head is included in the body code from Snondet to Sdet.

A det test block can contain all code of the head, including any bindings. If the tests succeed, only the code in the body which comes after the head needs to be done. Therefore a jump to Sdet is done, to avoid executing the same code twice.

\subsection{More details about the clause code}

The interaction of clause and procedure compilation is minimal. The procedure compiler only needs to know whether the clause occurs in one or more try-blocks, because this has an effect on the method of determining the failure addresses Fnocp and Fcp.

In ordinary WAM code, it is necessary to set up an environment with the allocate instruction before accessing permanent variables. A simple modification of the instruction set makes it possible to use permanent variables in the head of a clause without having to execute allocate first. Permanent variables are normally accessed from the E register, which points to the current environment. If $\mathrm{E}$ is not available (allocate has not yet been executed), then it is still possible to access permanent variables by using the A register if it points correctly to the top of stack. This idea can be used to delay allocate until after the test blocks.

All registers and arguments of structures have a mode. The mode of a variable depends on the instruction in which it first occurs. The mode can change during execution of the head code, as the variable becomes more instantiated. This means that each instruction exists in several versions, annotated with the arguments' mode(s).

\subsection{An example of clause compilation}

To illustrate the above considerations, consider the clause:

$$
\begin{aligned}
& \text { :-mode( } i, i, o) \text {. } \\
& \operatorname{diff}\left(\left[\mathrm{u}+-\mathrm{v}, \mathbf{x},-\mathrm{du}+\_\mathrm{dv}\right)\right. \text { :- } \\
& \text { atom ( } \mathrm{x}), \text { !, } \\
& \text { diff }(-\bar{u},-x,-d u) \text {, } \\
& \operatorname{diff}(-\mathrm{v}, \ldots \mathrm{x}, \ldots \mathrm{dv}) \text {. }
\end{aligned}
$$

The final code generated (which corresponds to figures 1 and 2) is given in an instruction set which is a variation of [Warren 83]. It is self-explanatory in most cases. The save and restore instructions will save and restore registers. The calltest and get_struc_in instructions execute a test, but instead of doing a general fail operation they simply jump to a label upon failure. 


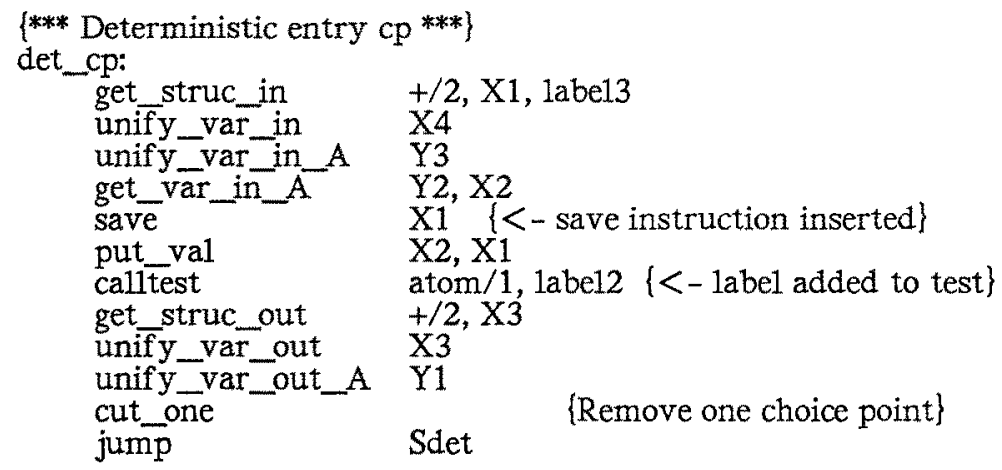

label2:

label1:

restore $\quad \mathrm{X} 1$

jump Fcp

\{*** Deterministic entry nocp $\left.{ }^{* * *}\right\}$

det_nocp:

(code identical to deterministic entry cp, except no cut_one) label4:

restore $\quad \mathrm{X} 1$

labell:

jump Fnocp

$\{* * *$ Nondeterministic entry $\mathrm{cp} * * *\}$

nondet_cp:

get_struc_in $+/ 2, \mathrm{X} 1$, 1abe15

labels: retry - Snondet else Fcp

jump Fcp

\{*** Nondeterministic entry nocp $* * *\}$

nondet_nocp:

(code analogous to nondet_cp)

$\{* * *$ Body code nondet entry ***\}

Snondet:

$\begin{array}{ll}\text { unify_var_in } & \text { X4 } \\ \text { unify_var_in_A } & \text { Y3 } \\ \text { get_var_in_A } & \text { Y2, X2 } \\ \text { put_val } & \text { X2,X1 } \\ \text { call_ } & \text { atom/1 } \\ \text { get_struc_out } & +/ 2, X 3 \\ \text { unify_var_out } & \text { X3 } \\ \text { unify_var_out_A } & \text { Y1 } \\ \text { cut_first } & \end{array}$




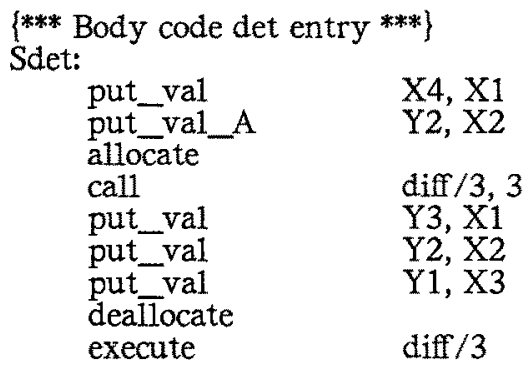

This example shows the kind of code explosion which is possible. Often a significant code reduction can be done by removing superfluous code. Such is the case here. For example, since this clause is deterministic the two nondet entries along with the nondet part of the body code can be removed.

\section{Compilation of a procedure}

The compilation of a procedure is done in two steps: (1) generation of the selection code and (2) generation of the try-blocks.

\subsection{The selection tree}

Information (e.g. mode) and assumptions about the actual arguments can be used to reduce the set of clauses to be tried. Different assumptions lead to different sets. Each set gives rise to a try-block. Selection of the proper try-block is done by the selection code which checks which assumptions are valid. Three kinds of information are used:

(1) The types (tags) and values of the actual arguments.

(2) The modes of the procedure.

(3) The head arguments of all the clauses.

The code which does the selection has the form of an n-ary tree: the selection tree. The tree has two kinds of nodes and one kind of leaf. The nodes do the selection. Both kinds of nodes will do a selection based on the contents of an argument register. The leaves try sequentially the sets of clauses which remain. Each branch of the tree has two attributes: the set of clauses still possible, and the set of argument registers yet to be considered.

(1) The switch node does a four-way branch depending on the type of an argument (variable, list, constant, or structure).

(2) The hash node does a multi-way branch depending on the value of an argument. The possible arguments are a series of constants or structures. The selection is done according to a hash table.

(3) Each leaf has one attribute: the set of clauses remaining after the selection.

The execution of a procedure call begins at the root of the selection tree. The root node does the first selection. Execution continues down the tree with further selection at the nodes until a leave is reached.

\subsubsection{A simple example of selection}

Consider the procedure:

$$
\begin{aligned}
& :-\operatorname{mode}(\mathrm{i}, \mathrm{i}) . \\
& \mathrm{h}(\mathrm{a}, \mathrm{x}):- \text { test, } 1, \ldots \\
& \mathrm{h}(\mathrm{a}, \mathrm{x}):-\ldots \\
& \mathrm{h}(\mathrm{b}, \mathrm{x}):-\ldots \\
& \mathrm{h}(\mathrm{b}, \mathrm{f}(3)):-\ldots
\end{aligned}
$$

The resulting code is: 


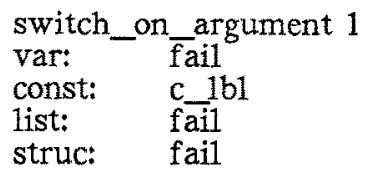

c__lbl:

hash_on_constant 1

$\mathrm{a} \quad \mathrm{a}-1 \mathrm{~b} 1$

a_1bl: $\quad\{$ try clauses 1 and 2$\}$

b_1b1: try_block $\quad$ C1,C2\} \{code for try-block explained later\}

switch_on_argument 2

var: - fail

const: clause3

list: clause3

struc: s_1bl

s_1bl:

try_block $\quad\{\mathrm{C} 3, \mathrm{C} 4\}$

\subsection{The try-block}

\subsubsection{The structure of a try-block}

An entry of a clause can be a member of one or more try-blocks. If it occurs in only one try-block (a single occurrence clause) then fixed failure addresses $F c p$ and $F$ nocp can be compiled in the clause to try the next clause. If it occurs in more than one try-block (a multiple occurrence entry) then indirect jumps via a new global register (called $T$ ) must be compiled. The $T$ register points to a table of $F$ nocp- $F c p$ address pairs. Each try-block has its own table. A correct, but not optimal, place to increment $T$, is in the beginning of each clause.

A try-block may contain a mixture of single or multiple occurrence clauses. This gives no problems. The address table only needs entries for the multiple occurrence clauses. If most clauses are single occurrence then the address tables will be short.

A try-block may contain a mixture of deterministic and nondeterministic clauses. This also is easily handled: for deterministic clauses use the det entries of the clause and for nondeterministic clauses use the nondet entries.

The execution of a try-block starts with the nocp entry of the first clause. If it fails then a jump is done to the $c p$ or nocp entries of the following clause, depending on whether the choice point was created or not. The goal is to avoid creating the choice point as long as possible, by testing and failing in a simple way. As long as the choice point is not needed execution will jump from nocp entry to nocp entry. When a simple test and fail is not possible the choice point will be created. From then on the following clauses are entered through the $c p$ entries. This pattern of execution is shown in figure 3 . Execution starts at the nocp entry of clause 1, which is nondeterministic. Clause 3 is deterministic. All other clauses are nondeterministic.

A deterministic clause cannot create a choice point. If it is entered at the nocp entry, then the next clause will also be tried at the nocp entry. Therefore the goal of delaying the creation of a choice point as long as possible will be achieved better if there are many deterministic clauses in the try-block, and if the first clauses are deterministic. 


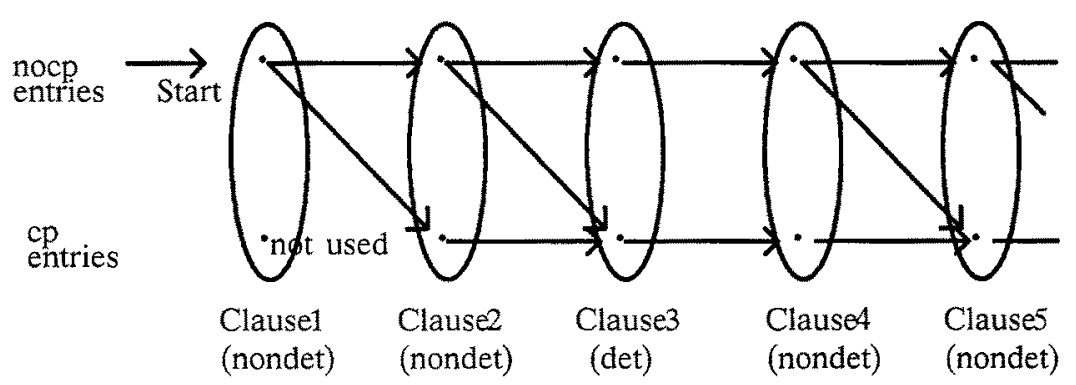

Figure 3 : Execution of a try-block

\subsubsection{Compilation scheme of the try-block}

The code for all try-blocks is generated together. This is necessary because clauses can be single or multiple occurrence. The existence of multiple occurrence clauses means that the try-blocks influence each other.

(1) Determine for each clause the number of try-blocks its nondet and det entries are in. This results in counts for nondet and det entries which must be kept separate.

(2) Generate code for each clause according to the scheme in section 3 . If the det entries are used in only one try-block then they are compiled with fixed failure addresses $F c p$ and $F$ nocp. If they occur in more than one try-block then indirect jumps via the $T$ register are compiled. The same is done for the nondet entries. This is the only interaction between procedure and clause compilation.

(3) For each try-block create a table of Fcp-Fnocp address pairs of all the multiple occurrence clauses in the try-block. The single occurrence clauses do not occur in the table.

(4) Generate code to load the $T$ register with the address of the table, and to jump to the first nocp entry in the table. For each try-block this results in code of the form:

$$
\begin{aligned}
& \text { start_try: \{Start of try-block's code\} } \\
& \text { move \#table_try, T } \\
& \text { jump nocp_clause1 } \\
& \text { table_try: } \quad \text { TTable of address pairs\} } \\
& \text { nocp_clause2, cp_clause2 } \\
& \text {... } \\
& \text { nocp_clauseN, last_clause } \\
& \text { fail, - trust_fail }
\end{aligned}
$$

The table starts with the second clause. The address of the first clause is in the jump instruction. The two special labels fail and trust fail have the following actions: fail will perform a general fail operation, trust fail will remove the choice point and then perform a general fail operation. 
The jump to a trust instruction is necessary for the last $c p$ entry. The trust removes the choice point and then the clause is entered at the nocp entry. This avoids the hole on the environment stack which exists if an environment is created before the choice point is removed. This hole can lead to stack overflows.

\subsection{An example of procedure compilation}

As an example procedure consider the following very small predicate:

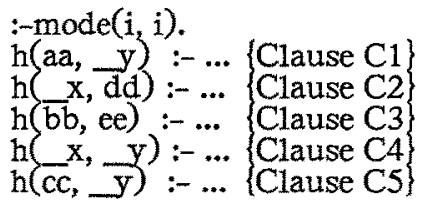

The procedure compilation of this predicate is done in two steps. The first step will give the selection tree which reduces the number of clauses to be tried. The second step compiles the try-blocks which try only those clauses. The selection code for this procedure is:

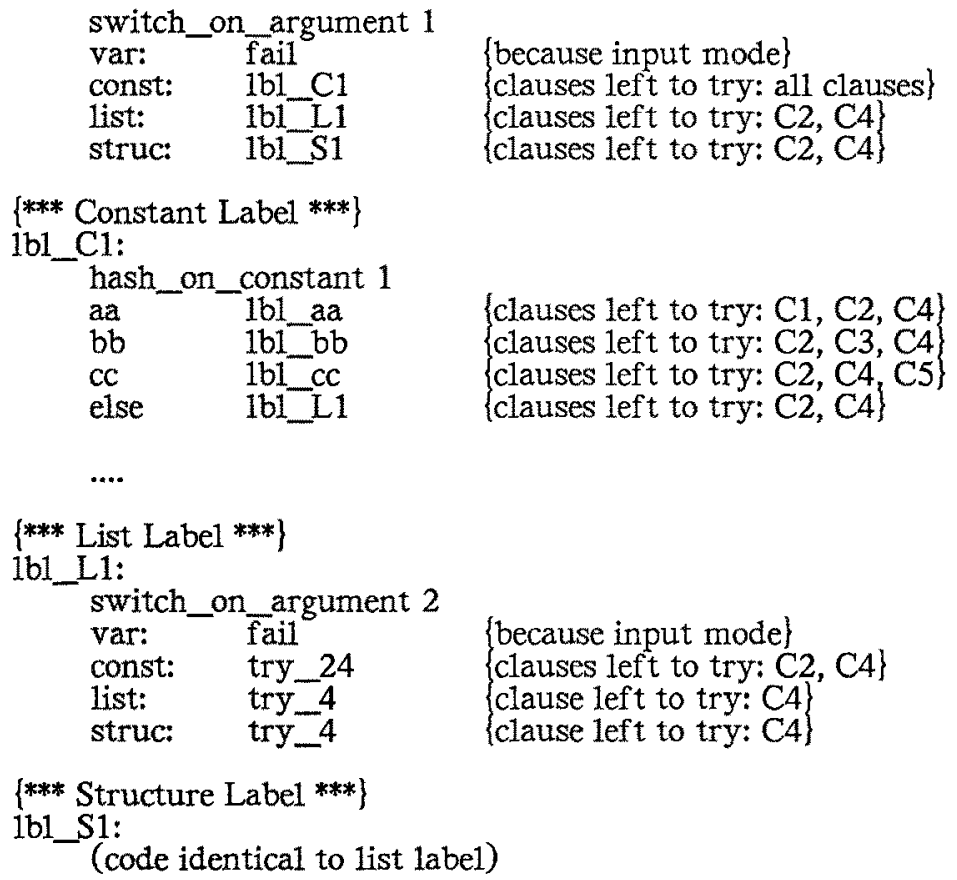

Only label 1b1_bb is worth expanding further, we leave it as an exercise. After the selection what remains are the following try-blocks:

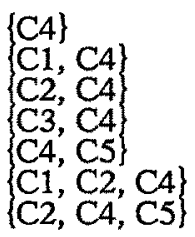

Now we must determine for each clause the number of try-blocks its det and nondet entries are in. This is easily found by inspection, giving: 
Clause C1: nondet entry occurs twice, as first clause.

Clause C2: $\quad$ nondet entry occurs twice as first and once as second clause.

Clause C3: nondet entry occurs once, as first clause.

Clause C4: $\quad$ nondet entry occurs twice, det entry five times.

Clause C5: $\quad$ det entry occurs twice, as last clause.

All the clauses' other det or nondet entries are not needed. Only clause C3 will be compiled with fixed failure addresses because it is the only single occurrence clause. All other clauses are compiled with indirection. Now we can combine what we know to generate the code for the try-blocks:

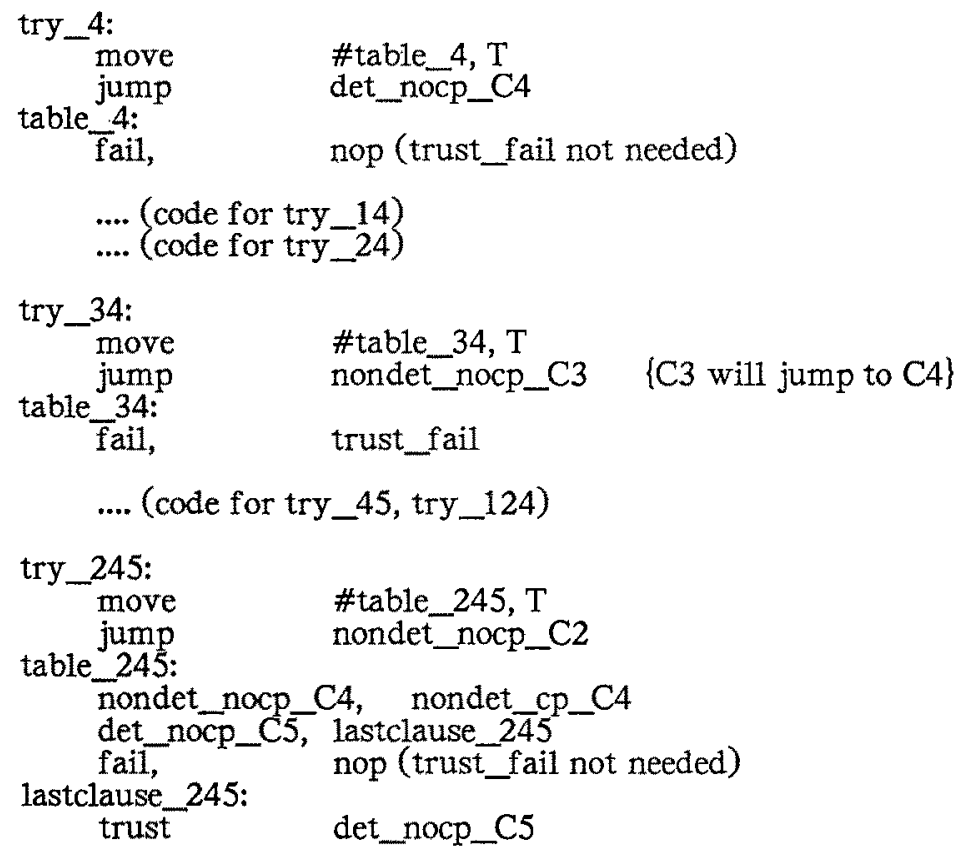

The code for the whole procedure consists of the selection code, the try-block code, and the clause code. This example shows a code multiplication similar to that of clause code. To reduce the amount of code it is possible to combine overlapping code segments in many places, for example the code segments which start with 1b1_L1 and 1b1_S1. Tryblocks which overlap in their last parts can also be combined.

\section{Evaluation of the optimizations}

In this section a number of simulated benchmark results are presented. The measurements are rough, but useful enough to decide whether the optimizations are worth the effort. The timings show that when the optimizations are applicable they can give significant performance improvements. We were not able to measure the speed improvement of all three optimizations taken together.

\subsection{Effects of determinism}

The speed improvement due only to the exploitation of deterministic clauses is measured. The main improvements of deterministic clauses are the elimination of choice point creation and general fail operations.

Total execution time is measured using the average times for each instruction. These times are taken from the PLM performance article [Dobry 85]. This gives a rough but useful indication of the speedup to be expected. 


\subsubsection{Results on the split/ 4 benchmark}

Measured was the total execution time of the procedure split/4 in Warren's quicksort benchmark [Warren 77]. The results are:

Speedup (unoptimized code has no environment): $\times 1.35$

Speedup (unoptimized code has an environment): $\times 1.43$

Number of machine cycles:

30260 for the old split (with environment),

28610 for the old split (without environment),

21230 for the new split.

The distinction between having or not having an environment is a consequence of the fact that in split/4 the cut flag has not been altered before the cut is encountered. Therefore no environment is needed for the cut. A smart compiler could recognize this and compile a special cut instruction which looks at the global cut flag instead of at the cut flag in the environment.

\subsubsection{Results on the minimum/3 benchmark}

Using the following definition:

$$
\begin{aligned}
& \text { min_list }([\mathrm{x}], \mathrm{x}) \text {. } \\
& \text { min_list }([\mathrm{xl}, \mathrm{1}, \mathrm{m}) \text { :- } \\
& \text { min list }(1,-y) \text {, } \\
& \text { minimum( } x, y, \ldots) \text {. }
\end{aligned}
$$

$:-\operatorname{mode}(\mathrm{i}, \mathrm{i}, \mathrm{o})$.

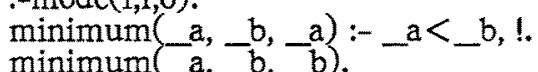

find the minimum of the 50-element list used in Warren's quicksort benchmark. The total execution time of minimum/3 is measured using the same method as for split/4. The result is a speedup of a factor of 2.6 .

Number of machine cycles:

3790 for the old minimum,

1470 for the new minimum.

No environment is needed for minimum/3. This is an extreme case in the sense that the bodies of the clauses are very small, so the elimination of try and fail will have a proportionally large effect. Therefore it gives an idea of the upper bound of the speedup.

\subsection{Effects of clause indexing}

The procedure borders from the Chat80 program [Warren 82] is improved by two orders of magnitude when clause selection is done on more arguments than just the first. Further measurements were not done for lack of time.

\subsection{Effects of modes}

The speed improvement due to modes alone (the speedup of unification), disregarding any other optimization made possible by modes is in the neighborhood of $10 \%$. This is an intuitive figure cited by the implementors of BIM-Prolog. More exact measurements were not done because of lack of time to disentangle the influence of modes on other optimizations. 


\section{Acknowledgements}

We would like to thank all the members of the Prolog research group at the university of Leuven for the congenial atmosphere. We thank especially Andre Marien and Alain Callebaut for many fruitful discussions and Maurice Bruynooghe for numerous suggestions to improve the final version of this paper.

This research was sponsored by grant KBAR/SOFT/1 from the Belgian 'Dienst voor Programmatie van het Wetenschapsbeleid' and by Defense Advanced Research Projects Agency (DOD) Arpa Order No. 4871, monitored by Space \& Naval Systems Warfare Command under Contract No. N00039-84-C-0089.

\section{References}

Bruynooghe M., Is logic programming real programming, in Pro. AIMSA86, eds. Ph. Jourrand, V. Sgurev, Vama, sept. 1986, North Holland, in print

Bruynooghe M., Weemeeuw P., Towards more efficiency of Prolog on conventional hardware, Report CW 45, Dept. of Computer Science, K.U.Leuven.

Bruynooghe M., Demoen B., Callebaut A. and Janssens G., Abstract interpretation at work, Draft report, Dept. of Computer Science, K.U.Leuven.

Debray S.K., Warren D.S., Detection and optimization of functional computations in Prolog, Proc. 3th Int. Conf. on Logic Programming., London, july 1986, 490-504.

Debray S.K., Warren D.S., Automatic mode inference for Prolog programs, Proc. 1986 Symposium on Logic Programming, Salt Lake City, sept 1986, 78-88.

Demoen, B., BIM-Prolog Manual, BIM, Kwikstraat 4, B-3078 Everberg Belgium, 1986.

Dobry, T. et al, Performance Studies of a Prolog Machine Architecture, Proc. 12th Int. Symp. Comp. Arch., June 1985.

Mellish, C.S., The Automatic Generation of Mode Declarations for Prolog Programs, Research Report 163, Dept. of Artificial Intelligence, Univ, of Edinburgh, 1981.

Mellish, C.S., Some Global Optimizations for a Prolog compiler, J. Logic Programming, pp. 43-66, Vol. 1, 1985.

Sawamura H., Takeshima T., Recursive unsolvability of determinacy, solvable cases of determinacy and their applications to Prolog optimization, Proc. 1985 Symposium on Logic Programming, Boston, july 1985, 200-207.

Van Roy, P., A Prolog Compiler for the PLM, Report No. UCB/CSD 84/203, Univ. of California, Berkeley, Nov. 1984.

Van Roy, P., Demoen, B., Improving the execution speed of compiled Prolog with modes, clause selection, and determinism. CW Report No. 51, K.U.Leuven, Dept. of Comp. Sc., nov. 1986.

Warren, D.H.D., Applied Logic - Its use and implementation as a programming tool, Ph.D. Thesis, Univ. of Edinburgh, 1977. Reprinted as Technical Note 290, SRI International, 1983.

Warren, D.H.D., An Efficient Easily Adaptable System for Interpreting Natural Language Queries, American Journal of Computational Linguistics, pp. 110-122, Vol. 8, 1982.

Warren, D.H.D., An Abstract Prolog Instruction Set, AI Center, SRI International, Menlo Park CA 94025, 1983. 